\title{
SWARM DIRECTIONS EMBEDDED DIFFERENTIAL EVOLUTION FOR FASTER CONVERGENCE OF GLOBAL OPTIMIZATION PROBLEMS
}

\author{
MUSRRAT ALI \\ Department of Computer Science, Sungkyunkwan University \\ Suwon 440-746, Republic of Korea \\ musrrat.iitr@gmail.com \\ MILLIE PANT \\ Department of Paper Technology, Indian Institute of Technology Roorkee \\ Roorkee, Uttarakhand 247667 , India \\ millifpt@iitr.ernet.in \\ AJITH ABRAHAM \\ Center of Excellence for Quantifiable Quality of Service \\ Norwegian University of Science and Technology, Norway \\ ajith.abraham@ieee.org \\ CHANG WOOK AHN \\ Department of Computer Science, Sungkyunkwan University \\ Suwon 440-746, Republic of Korea \\ cwan@skku.edu
}

\begin{abstract}
In the present study we propose a new hybrid version of Differential Evolution (DE) and Particle Swarm Optimization (PSO) algorithms called Hybrid DE or HDE for solving continuous global optimization problems. In the proposed HDE algorithm, information sharing mechanism of PSO is embedded in the contracted search space obtained by the basic DE algorithm. This is done to maintain a balance between the two antagonist factors; exploration and exploitation thereby obtaining a faster convergence. The embedding of swarm directions to the basic DE algorithm is done with the help of a "switchover constant" called $\alpha$ which keeps a record of the contraction of search space. The proposed HDE algorithm is tested on a set of 10 unconstrained benchmark problems and four constrained real life, mechanical design problems. Empirical studies show that the proposed scheme helps in improving the convergence rate of the basic DE algorithm without compromising with the quality of solution.
\end{abstract}

Keywords: Differential evolution; particle swarm optimization; hybridization; global optimization.

\section{Introduction}

Optimization problems arise in various disciplines such as engineering designs, agricultural sciences, manufacturing systems, economics, physical sciences, pattern recognition etc. in fact optimization techniques are being extensively used in various 
spheres of human activities, where decisions have to be taken in some complex situation which can be represented by mathematical models. Optimization can thus be viewed as a kind of decision making, or more specifically, as one of the major quantitative tools in network of decision making, in which decisions have to be taken to optimize one or more objectives under some prescribed set of circumstances. Significant amount of work has been done in the field optimization which mainly revolves around developing efficient methods for solving mathematical models of optimization problems. Some of the classical optimization techniques include Methods Linear Programming (LP) and traditional nonlinear (NL) techniques like Bellman's principle, Lagrange's multiplier method, quadratic programming approach etc. A detailed study of classical optimization methods can be found in Refs. 28, 8 and 43. While LP approaches are restricted to linear models, the problem with most of the traditional NL techniques is that they are gradient based and hence cannot be applied for finding out the solution to the problems which have a rough or discontinuous surface. Consequently, researchers working in the field of optimization have laid much attention on a class of derivative free techniques that can be applied for solving complex optimization models.

One class of derivative free techniques consists of nature inspired evolutionary search algorithms. These techniques have gained a lot of popularity in the recent years because of their ability to deal with complex optimization problems which are otherwise difficult to solve using traditional methods. Some well known evolutionary/nature inspired algorithms are Genetic Algorithms, ${ }^{25}$ Evolutionary Programming, ${ }^{24}$ Evolutionary Strategies $^{7}$ Particle Swarm optimization ${ }^{43}$ and Differential Evolution ${ }^{51}$ etc. The working of all these algorithms is based on some biological or social metaphor.

Out of these, PSO and DE are comparatively newer than others. These are stochastic, population based search techniques and have been applied successfully to a wide range of problems occurring in various disciplines. ${ }^{2-4,10,16-18,22,23,26,35,36,41,44,45,52,54,55,58,60}$ A number of variants of these algorithms have been developed in the past decade to improve their performance. $^{5,9,29,42,46,49,50,57}$

One class of modification consists of integrating DE and PSO algorithms where the advantages of both the algorithms are blended together to form a new algorithm. DE has the advantage of not being biased towards any prior defined distribution for sampling mutational step sizes and its selection operator follows a hill-climbing process. According to the literature, the DE algorithm performs very well in the initial stages however as the search proceeds, the convergence rate of DE slows down gradually.

On the basis of practical experience, researchers have shown that DE may sometimes stop proceeding toward the global optimum even though the population has not converged to a local optimum or any other point. ${ }^{34}$ There is a possibility that the new points may enter the population but the algorithm does not show any progress in finding any better solutions. This situation is termed as stagnation. DE also suffers from the problem of premature convergence, where the population converges to some local optima of a multimodal objective function, losing its diversity. The probability of stagnation depends on how many different potential trial solutions are available and also on their capability to enter into the population of the subsequent generations. ${ }^{34}$ 
Further, like other evolutionary computing algorithms, the performance of DE deteriorates with the growth of the dimensionality of the search space as well. A good volume of work is devoted in the direction of improvement of DE algorithm. These modifications may be differentiated as variation in the probabilities of crossover and mutation, development of new mutation or crossover operators, development of adaptive parameters $1,5,9,12,19,29,34,37,39,40,42,46,57,59$ etc. Hybridization of DE with some local search technique or with some stochastic technique can be found in Refs. 11, 14, 27, 30-32, 38, $53,56,61$ and 62.

The idea behind hybridization is to merge the positive features of two (or more than two) algorithms in order to obtain a new algorithm which is better than the two parent algorithms which are used for hybridization. In the present study we propose a hybrid version of DE and PSO called hybridized DE or HDE. The proposed HDE algorithm works in two phases; the DE phase (or the first phase) and the PSO phase (or the second phase). The change of phases is decided according to a switchover constant $\alpha$. By embedding the information sharing mechanism of PSO in the later stages of the search we try to push the particles towards a local attractor which is hopefully near the global optimum.

The remaining paper is organized as follows: In Sec. 2, we give a brief overview of the hybridized DE-PSO algorithms. In Sec. 3, basic DE and PSO algorithms are described. The proposed HDE algorithm is given in Sec. 4. Benchmark problems and experimental settings are given in Sec. 5 and numerical results are given in Sec. 6 . Finally, the conclusions based on the present study are drawn in Sec. 7.

\section{A Brief Literature Review on Earlier Work Done}

As mentioned in the previous section, both DE and PSO have undergone a plethora of changes since their development in 1995. However, not much instances are available in literature which combines the features of DE and PSO together in a single algorithm. In this section we give a brief review of some earlier work in which the two algorithms are merged together.

Hendtlass ${ }^{47}$ used the DE perturbation approach to adapt particle positions. In his algorithm, named SDEA, particles' positions are updated only if their offspring have better fitness. The DE reproduction process is applied to the particles in swarm at specified intervals. At the specified intervals, the PSO swarm serves as the population for $\mathrm{DE}$ algorithm, and the DE is executed for a number of generations. After execution of DE, the evolved population is further optimized using PSO. Hendtlass applied his algorithm SDEA on four unconstrained benchmark problems for different dimensions and for different population sizes.

Zhang and $\mathrm{Xie}^{62}$ used different techniques in random, rather than combining them, in their Differential Evolution (DE) PSO (DEPSO). In this case the DE and canonical PSO operators were used on alternate generations; when DE was in use, the trial mutation replaced the individual best at a rate controlled by a crossover constant and a random dimension selector that ensured at least one mutation occurred each time. 
Kannan et al..$^{20}$ applied DE to each particle for a finite number of iterations, and replaced the particle with the best individual obtained from the DE process.

Talbi and Batauche ${ }^{53}$ proposed an algorithm named DEPSO. It differs from the DEPSO algorithm proposed by Zhang and Xie as the DE operators are applied only to the best particle obtained by PSO. They applied their algorithm on medical image processing problem.

In Hao et al.' $\mathrm{s}^{63}$ hybrid version, which is also named as DEPSO, the candidate solution is generated either by DE or by PSO according to some fixed probability distribution. They applied their algorithm for solving unconstrained global optimization problems.

Das et al. ${ }^{18}$ proposed a scheme of adjusting velocities of the particles in PSO with a vector differential operator borrowed from the DE family. In their proposed PSO-DV algorithm, they omitted the cognitive term of the canonical PSO and updated the particle velocities by a new term containing the weighted difference (inspired by DE mutation scheme) of the position vectors of any two distinct particles randomly chosen from the swarm. They also applied their algorithm on a test suite of selected unconstrained benchmark problems.

Omran et al. ${ }^{38}$ proposed a hybrid version of Bare Bones PSO and DE called it BBDE. In their approach, they combined the concept of barebones PSO with self adaptive DE strategies. The mutation operator of DE is used to explore around the current attractor by adding a difference vector to the attractor. Crossover is done with randomly selected personal best as these personal bests represent a memory of the best solution found by individuals since the start of the search process. They validated their algorithm on a set of unconstrained benchmark problems and also applied to image classification problem.

The work of Jose et al. ${ }^{64}$ evaluated a Particle Swarm Optimizer hybridized with Differential Evolution and applied it to the Black-Box Optimization Benchmarking for noisy functions. Their algorithm was once named as DEPSO algorithm. In this version of DEPSO, the differential variation schemes of DE are used for updating the velocities of the swarm particles.

Zhang et al. ${ }^{61}$ developed a hybrid of DE and PSO called DE-PSO, where three alternative updating strategies are used. The DE updating strategy is executed once in every $l$ generations and if a particle's best encountered position and the position of its current best neighbor are equal then random updating strategy is executed otherwise PSO updating strategy is used.

Liu et al. ${ }^{65}$ proposed a novel hybrid algorithm named PSO-DE, in which DE is incorporated to update the previous best positions of PSO particles to force them to jump out of local attractor in order to prevent stagnation of population.

Copanio et al. ${ }^{66}$ developed a Superfit Memetic Differential Evolution (SFMDE) by hybridizing DE and PSO with two other local search methods; Nelder Mead algorithm and Rosenbrock algorithm. In SFMDE, PSO assists DE in the beginning of the optimization process to generate a "super-fit individual", the local searches are then applied adaptively by means of a parameter which measures the quality of super-fit 
Table 1. A summary of hybridized PSO + DE algorithms.

\begin{tabular}{|c|c|c|c|}
\hline Author/s & Year & $\begin{array}{l}\text { Name of the } \\
\text { Algorithm }\end{array}$ & Application/s \\
\hline Hentdlass $^{47}$ & 2001 & SDEA & Unconstrained global optimization \\
\hline Zhang and $\mathrm{Xie}^{62}$ & 2003 & DEPSO & Unconstrained global optimization \\
\hline Kannan et al. ${ }^{20}$ & 2004 & & Generation Expansion planning \\
\hline Talabi and Batouche ${ }^{53}$ & 2004 & DEPSO & Medical Image Processing \\
\hline Hao et al. ${ }^{63}$ & 2007 & DEPSO & Unconstrained global optimization \\
\hline Das et al. ${ }^{18}$ & 2008 & PSO-DV & Engineering Design \\
\hline Omran et al..$^{38}$ & 2008 & BBDE & $\begin{array}{l}\text { Unconstrained Optimization Problems and Image } \\
\text { Processing }\end{array}$ \\
\hline Jose et $a l .{ }^{64}$ & 2009 & DEPSO & Noisy functions \\
\hline Zhang et al. ${ }^{61}$ & 2009 & DE-PSO & Unconstrained Optimization \\
\hline Liu et $a l .{ }^{65}$ & 2009 & PSO-DE & Constrained optimization and engineering problems \\
\hline Caponio et al. ${ }^{66}$ & 2009 & SFMDE & $\begin{array}{l}\text { Unconstrained global optimization and engineering } \\
\text { design problems }\end{array}$ \\
\hline $\mathrm{Xu}$ and $\mathrm{Gu}^{67}$ & 2009 & PSOPDE & Unconstrained global optimization \\
\hline Pant et al. ${ }^{68}$ & 2009 & DE-PSO & Unconstrained global optimization \\
\hline Khamsawang et al. ${ }^{69}$ & 2010 & PSO-DE & Power Systems \\
\hline
\end{tabular}

individual. SFMDE was applied for solving unconstrained standard benchmark problems and two engineering problems.

$\mathrm{Xu}$ and $\mathrm{Gu}^{67}$ proposed Particle Swarm Optimization with prior crossover differential evolution (PSOPDE). Their version is distinct from other PSO-DE hybrids in three ways; (1) the particles in the swarm are not just led towards global and personal best positions but also depend on the average position and velocity of the particles (2) DE is integrated with PSO for local search and (3) a new crossover operation between the target and an extra population is implemented before the DE component. They applied their algorithm for solving five unconstrained benchmark problems.

Khamsawang et al. ${ }^{69}$ proposed an improved hybrid algorithm based on conventional particle swarm optimization and differential evolution (called PSO-DE) for solving an economic dispatch(ED) problem with the generator constraints. In PSO-DE, the mutation operators of the differential evolution are used for improving diversity exploration of PSO and are activated if velocity values of PSO are near to zero or violate the boundary conditions.

The DE-PSO algorithm suggested by Pant et al. ${ }^{68}$ starts like the usual DE algorithm. it enters the PSO phase if the optimality criteria are not met by the DE algorithm. They tested their algorithm on a set of unconstrained benchmark problems. A brief summary of hybrid PSO and DE algorithms is given in Table 1.

\section{Differential Evolution (DE) and Particle Swarm Optimization (PSO)}

DE was proposed by Storn and Price ${ }^{51}$ in 1995. It soon became a popular tool for solving global optimization problems because of several attractive features like having fewer 
control parameters, ease in programming, efficiency etc. DE is similar to GAs in the sense that it uses same evolutionary operators like mutation, crossover and selection for guiding the population towards the optimum solution. Nevertheless, it's the application of these operators that makes DE different from GA. The main difference between GAs and DE is that; in GAs, mutation is the result of small perturbations to the genes of an individual while in DE mutation is the result of arithmetic combinations of individuals. Also in DE, mutation plays a prominent role whereas, in GA, crossover is the major operator. At the beginning of the evolution process, the mutation operator of DE favors exploration. As evolution progresses, the mutation operator favors exploitation. Hence, DE automatically adapts the mutation increments (i.e. search step) to the best value based on the stage of the evolutionary process. Mutation in DE is therefore not based on a predefined probability density function. Moreover, GA may work even without the presence of a mutation operator as crossover is the prime operator of GA but the main component of DE is the generation of mutant vector. Also in DE mutation is applied before crossover whereas in GA, mutation is always applied after crossover. Throughout the study we shall consider the mutation strategy DE/rand/1/bin also known as the classical version of DE. The three main equations used in DE are given as follows:

$$
\begin{gathered}
U_{i, G+1}=X_{r 3, G}+F^{*}\left(X_{r 1, G}-X_{r 2, G}\right) \\
t_{j, i . G+1}= \begin{cases}u_{j, i . G+1} & \text { if } r \text { and } j \leq C_{r} \vee j=k \\
x_{j, i . G} & \text { otherwise }\end{cases} \\
X_{i \cdot G+1}= \begin{cases}T_{i . G+1} & \text { if } f\left(T_{i . G+1}\right) \leq f\left(X_{i . G}\right) \\
X_{i, G} & \text { otherwise }\end{cases}
\end{gathered}
$$

Equations (1), (2) and (3) represent the mutation, crossover and selection operations respectively, for $\mathrm{DE}$. $\mathrm{F}$ is the scaling factor and $\mathrm{Cr}$ is the probability of crossover.

PSO is a multi-agent parallel search technique developed by Kennedy and Eberhart in $1995,{ }^{33}$ inspired by social behavior of bird flocking or fish schooling. The particles or members of the swarm fly through a multidimensional search space looking for a potential solution. Each particle adjusts its position in the search space from time to time according to the flying experience of its own and of its neighbors (or colleagues). Each particle maintains a memory of its previous best position and also the best position among all the particles. During each generation each particle is accelerated toward the particles previous best position and the global best position. At each iteration a new velocity value for each particle is calculated based on its current velocity, the distance from the global best position. The new velocity value is then used to calculate the next position of the particle in the search space. This process is then iterated a number of times or until a minimum error is achieved.

$$
\begin{gathered}
V_{i}^{G+1}=w^{G} V_{i}^{G}+c_{1} r_{1}\left(P_{i}^{G}-X_{i}^{G}\right)+c_{2} r_{2}\left(P_{\mathrm{best}}^{G}-X_{i}^{G}\right) \\
X_{i}^{G+1}=X_{i}^{G}+V_{i}^{G+1}
\end{gathered}
$$




$$
w^{G}=\left(w_{\max }-w_{\min }\right) \times \frac{(\text { maxiteration-Iteration })}{\text { Iteration }}+w_{\min }
$$

Equations (4) and (5) represent the velocity and position equations of the swarm particles. Equation (6) gives the inertia weight taken for the present study. $P_{i}^{G}$ represents the personal best position of the particle and $P_{\text {best }}^{G}$ represents the global best particle. $c_{1}, c_{2}$ represents the acceleration constants and $r_{1}, r_{2}$ are uniformly distributed random numbers in the range $(0,1)$.

\section{Proposed Hybrid Differential Evolution (HDE)}

In hybridized algorithms it is very crucial to decide how the algorithms should be merged in order to get the maximum benefit. In the proposed HDE algorithm, the initial search space is contracted using the DE algorithm and then PSO is applied to further refine the search space with the hope of finding the global optimal solution. The change of DE phase to PSO phase is decided with the help of a switchover constant denoted by $\alpha$. The final contracted population obtained by DE is arranged in ascending order of fitness function values and upper half of the population undergoes the PSO operation i.e. to say that PSO is applied on only half of the population consisting of elite solutions. The working of HDE can be explained with the help of the following steps.

Step 1. Generate randomly NP vectors, each of $n$ dimensions:

$X_{i, j}=X_{\min , j}+\operatorname{rand}(0,1)\left(X_{\max , j}-X_{\min , j}\right)$, where $X_{\min , j}$ and $X_{\max , j}$ are lower and upper bounds for $j$ th component respectively, $\operatorname{rand}(0,1)$ is a uniformly distributed random number between 0 and 1 .

Step 2. Calculate the objective function value $f\left(X_{i}\right)$ for all $X_{i}$.

Step 3. Corresponding to target vector $X_{i}$ select three distinct points from population and generate perturbed individual $U_{i}$ using equation (1).

Step 4. Recombine the each target vector $X_{i}$ with perturbed individual generated in step 3 to generate a trial vector $T_{i}$ using equation (2).

Step 5. Check whether each variable of the trial vector is within range. If not keep it within range using $T_{i, j}=2 * X_{\min , j}-T_{i, j}$, if $T_{i, j}<X_{\min , j}$ and $T_{i, j}=2 * X_{\max , j}-T_{i, j}$, if $T_{i, j}>X_{\max , j}$, otherwise go to Step 6 .

Step 6. Calculate the objective function value for vector $T_{i}$.

Step 7. Choose better of the two (function value at target and trial point) using equation (3) for next generation.

Step 8. Check whether $\left|f_{\max }-f_{\min }\right|<\alpha$ (given threshold) value. If yes then switchover to PSO (Step 10); otherwise go to Step 9.

Step 9. Check whether maximum number of function evaluation has been completed. If yes, stop; otherwise go to Step 3.

Step 10. Sort the final population obtained by DE algorithm in ascending order. Take first NP/2 individuals as initial swarm; assign initial velocity zero, personal best position same as particle position and global best position particle position having minimum function value. 
Step 11. Update velocity $V_{i}^{G+1}$ and position $X_{i}^{G+1}$, according to equations (4) and (5) of all NP/2 particles.

Step 12. Evaluate objective function value $f\left(X_{i}^{G+1}\right)$ for all $X_{i}^{G+1}$.

Step 13. Update particle best position: if $f\left(X_{i}^{G+1}\right)<f\left(P_{i}\right)$ then $f\left(P_{i}\right)=f\left(X_{i}^{G+1}\right)$ and $P_{i}=$ $X_{i}^{G+1}$.

Step 14. Find global best $P_{\text {best }}$ position of particle and update inertia weight.

Step 15. Check whether convergence criterion is met. If yes, stop: otherwise go to Step 16.

Step 16. Check whether maximum number of function evaluation has been completed. If yes, then stop; otherwise go to Step 11 .

The parameter $\alpha$ defined in Step 8, plays an important role in HDE. It keeps a track of the contraction of the search space in order to initiate the PSO algorithm.

\section{Benchmark Problems and Experimental Settings}

\subsection{Benchmark and real life problems}

To analyze the performance of the proposed HDE algorithm we tested it on 10 unconstrained standard bench mark problems taken from literature. All these problems are scalable in nature i.e. their dimension can be varied in order to increase their complexity. We have tested these problems for dimensions 10 and 30. The mathematical model of the problems along with the true global minimum is given the Appendix A.

To further analyze the efficiency of HDE we used it for solving four real life mechanical design problems. All the four problems are constrained in nature. The constraints are dealt with by using the tournament based approach proposed by Deb $^{21}$ for solving constrained optimization problems. According to this rule:

- Between two feasible solutions, the one with the highest fitness value wins.

- If one solution is feasible and the other one is infeasible, the feasible solution wins.

- If both solutions are infeasible, the one with the lowest sum of constraint violation is preferred.

This method does not require a penalty factor as the selection procedure only performs pair wise comparisons. The feasible solutions therefore have fitness equal to their objective function value, and the use of constraint violation in the comparisons aims to push the infeasible solutions towards the feasible region.

\subsubsection{Experimental settings}

As discussed in Sec. 4, HDE starts with the usual DE algorithm for which, the lower limit for population size, NP, is 4 since the mutation process requires at least three other chromosomes for each parent. While testing the algorithms, we began by using the optimized control settings of DE. Population size, NP can always be increased to help maintain population diversity. As a general rule, an effective NP is between $3 * \mathrm{n}$ and 
$10 * \mathrm{n}$, but can often be modified depending on the complexity of the problem. For the present study we performed several experiments with the population size as well as with the crossover rate and mutation probability rate and observed that for problems up to dimension 30 a population size of $10 * \mathrm{n}$ is sufficient. Values of scale $\mathrm{F}$, outside the range of 0.4 to 1.2 are rarely effective and $F=0.5$ can be considered to be a good initial choice. In general higher value of $C_{r}$ help in speeding up the convergence rate therefore in the present study we have taken $C_{r}=0.5$.

HDE also contains an additional parameter $\alpha$, which keeps a track of the contraction of search space and decides the initiation of PSO algorithm. We observed the preformance of the proposed HDE algorithm for three values of $\alpha ; 0.5,0.05$ and 0.005 .

The PSO algorithm in HDE is applied to a region which has already contrcted i.e. to say the PSO algorithm is applied to a small search space (hopefully) near the global minima. Therefore, we have taken the inertia weight $w$ which decrease uniformly from 0.4 to 0.2 . Also in order to give more weight to the global best position, the acceleration coeffient $C_{1}$ and $C_{2}$ are taken as 0.5 and 2.0 respectively. All the algorithms are executed on a PIV PC, using DEV $\mathrm{C}++$, thirty times for each problem. Random numbers are generated using the inbuilt random number generator rand ( ) function available in DEVC++.

In every case, a run was terminated when the best function value obtained is less than a threshold $\left(10^{-4}\right)$ for the given function or when the maximum number of function evaluation (NFE $=10^{6}$ ) was reached. In order to have a fair comparison, these settings are kept the same for all algorithms over all benchmark functions during the simulations.

\section{Numerical Results}

\subsection{Numerical results for unconstrained benchmark problems}

The performance of the proposed HDE algorithm, for different values of $\alpha$, is analyzed on a set of ten unconstrained benchmark problems and the numerical results are compared with the traditional DE algorithm. The performance measures used for comparison include average fitness function value, standard deviation (Std), average numbers of functions evaluations and average CPU time taken. The corresponding results are given in Tables 2-5.

Under the given parameter settings, it can be seen that the traditional DE was not able to achieve the desired accuracy of $10^{-4}$ in case of $f_{R B}$ (Rosenbrock function) whereas, for $f_{G W}$ (Griewanks function) HDE was not able to achieve the desired accuracy for $\alpha=0.5$. For the remaining values of $\alpha(=0.05$ and 0.005$)$ HDE was able to achieve the desired accuracy for all the test cases.

From the numerical results it can be seen that although in terms of average fitness function value, the proposed HDE algorithm does not show much improvement in comparison to the basic DE, but in terms of NFE and CPU time taken, the proposed HDE algorithm performs much better than the basic DE algorithm. If discuss the sensitivity of 
Table 2. DE versus HDE (for $\alpha=0.5,0.05,0.005$ ) in terms of average fitness function value and standard deviation (Std) for unconstrained benchmark problems of dimension 10.

\begin{tabular}{|c|c|c|c|c|}
\hline \multirow{2}{*}{ Fun } & DE & HDE $(\alpha=0.5)$ & HDE $(\alpha=0.05)$ & HDE $(\alpha=0.005)$ \\
\cline { 2 - 5 } & Fitness (Std) & Fitness (Std) & Fitness (Std) & Fitness (Std) \\
\hline \multirow{2}{*}{$f_{\text {SP }}$} & $1.40711 \mathrm{e}-05$ & $\mathbf{5 . 6 2 6 3 0 e - 0 6}$ & $1.22108 \mathrm{e}-05$ & $8.8269 \mathrm{e}-06$ \\
& $(5.17314 \mathrm{e}-06)$ & $(3.91721 \mathrm{e}-06)$ & $(7.65373 \mathrm{e}-06)$ & $(5.16438 \mathrm{e}-06)$ \\
\hline \multirow{2}{*}{$f_{\text {ACK }}$} & $5.94765 \mathrm{e}-05$ & $3.56962 \mathrm{e}-05$ & $5.44005 \mathrm{e}-05$ & $\mathbf{3 . 4 3 1 1 3 e - 0 5}$ \\
& $(1.0848 \mathrm{e}-05)$ & $(1.30165 \mathrm{e}-05)$ & $(2.94382 \mathrm{e}-05)$ & $(1.11886 \mathrm{e}-05)$ \\
\hline \multirow{2}{*}{$f_{R G}$} & $1.13561 \mathrm{e}-05$ & $9.00661 \mathrm{e}-06$ & $1.16375 \mathrm{e}-05$ & $\mathbf{6 . 8 3 0 9 5 e - 0 6}$ \\
& $(3.38399 \mathrm{e}-06)$ & $(5.72322 \mathrm{e}-06)$ & $(7.7549 \mathrm{e}-06)$ & $(5.40267 \mathrm{e}-06)$ \\
\hline \multirow{2}{*}{$f_{R B}$} & & $\mathbf{9 . 0 2 5 2 6 e - 1 4}$ & 0.00096 & 0.00063 \\
& & $(0.00340)$ & $(0.00205)$ & $(0.00064)$ \\
\hline \multirow{2}{*}{$f_{G W}$} & $1.46893 \mathrm{e}-05$ & & $2.74695 \mathrm{e}-06$ & $\mathbf{1 . 0 3 9 2 6 e - 0 5}$ \\
& $(3.77339 \mathrm{e}-06)$ & - & $(8.24084 \mathrm{e}-06)$ & $(5.69282 \mathrm{e}-06)$ \\
\hline \multirow{2}{*}{$f_{L M 2}$} & $1.60214 \mathrm{e}-05$ & $9.83868 \mathrm{e}-006$ & $\mathbf{7 . 9 6 4 1 3 e - 0 6}$ & $8.12357 \mathrm{e}-06$ \\
& $(4.07044 \mathrm{e}-06)$ & $(8.48897 \mathrm{e}-06)$ & $(5.48033 \mathrm{e}-06)$ & $(5.08711 \mathrm{e}-06)$ \\
\hline \multirow{2}{*}{$f_{p p}$} & -45.77850 & -45.77850 & -45.77850 & -45.77850 \\
& $(4.25827 \mathrm{e}-06)$ & $(4.24994 \mathrm{e}-06)$ & $(6.03048 \mathrm{e}-06)$ & $(2.57806 \mathrm{e}-06)$ \\
\hline \multirow{2}{*}{$f_{s i s}$} & -3.49998 & -3.49999 & -3.49999 & -3.49999 \\
& $(4.62082 \mathrm{e}-06)$ & $(9.00903 \mathrm{e}-06)$ & $(7.38833 \mathrm{e}-06)$ & $(4.48909 \mathrm{e}-06)$ \\
\hline \multirow{2}{*}{$f_{s t}$} & 0.00000 & 0.00000 & 0.00000 & 0.00000 \\
& $(0.00000)$ & $(0.00000)$ & $(0.00000)$ & $(0.00000)$ \\
\hline \multirow{2}{*}{$f_{S W F}$} & 0.00024 & 0.00023 & 0.00023 & 0.00023 \\
& $(4.14499 \mathrm{e}-06)$ & $(4.76434 \mathrm{e}-06)$ & $(1.35658 \mathrm{e}-05)$ & $(5.46631 \mathrm{e}-06)$ \\
\hline
\end{tabular}

Table 3. DE versus HDE (for $\alpha=0.5,0.05,0.005$ ) in terms of average fitness function value and standard deviation (Std.) for unconstrained benchmark problems of dimension 30.

\begin{tabular}{|c|c|c|c|c|}
\hline \multirow{2}{*}{ Fun } & DE & HDE $(\alpha=0.5)$ & HDE $(\alpha=0.05)$ & HDE $(\alpha=0.005)$ \\
\cline { 2 - 5 } & Fitness (Std) & Fitness $($ Std $)$ & Fitness $($ Std $)$ & Fitness $($ Std $)$ \\
\hline \multirow{2}{*}{$f_{\text {SP }}$} & $4.96717 \mathrm{e}-05$ & $\mathbf{2 . 3 8 6 7 9 e - 0 5}$ & $9.01265 \mathrm{e}-05$ & $6.02874 \mathrm{e}-05$ \\
& $7.77918 \mathrm{e}-06$ & $3.29303 \mathrm{e}-05$ & $4.05859 \mathrm{e}-05$ & $1.67625 \mathrm{e}-05$ \\
\hline \multirow{2}{*}{$f_{\text {ACK }}$} & $\mathbf{0 . 0 0 0 1 4}$ & 0.00047 & 0.00024 & 0.00018 \\
& $1.66159 \mathrm{e}-05$ & 0.00061 & 0.00011 & $8.38164 \mathrm{e}-05$ \\
\hline \multirow{2}{*}{$f_{R G}$} & 105.858 & 104.131 & $\mathbf{8 7 . 8 9 0 4}$ & 89.1484 \\
& 6.16943 & 6.20312 & 6.01522 & 5.22234 \\
\hline \multirow{2}{*}{$f_{R B}$} & 15.1913 & $\mathbf{2 . 6 5 0 9 7 e - 1 5}$ & 13.6827 & 13.8989 \\
& 1.70576 & $1.55107 \mathrm{e}-10$ & 1.00897 & 1.63056 \\
\hline \multirow{2}{*}{$f_{G W}$} & $5.07342 \mathrm{e}-05$ & 0.00744 & $\mathbf{4 . 4 3 1 0 5 e - 0 5}$ & $5.31735 \mathrm{e}-05$ \\
& $5.83072 \mathrm{e}-06$ & 0.00084 & $4.57603 \mathrm{e}-05$ & $1.42185 \mathrm{e}-05$ \\
\hline \multirow{2}{*}{$f_{L M 2}$} & $\mathbf{4 . 7 3 7 2 5 e - 0 5}$ & 0.00031 & $6.89487 \mathrm{e}-05$ & $5.45789 \mathrm{e}-05$ \\
& $9.83696 \mathrm{e}-06$ & 0.00041 & $2.48868 \mathrm{e}-05$ & $2.62944 \mathrm{e}-05$ \\
\hline \multirow{2}{*}{$f_{p p}$} & -997867.0 & -997867.0 & -997867.0 & -997867.0 \\
& $2.45355 \mathrm{e}-06$ & $3.341784 \mathrm{e}-05$ & $3.22517 \mathrm{e}-05$ & $1.31195 \mathrm{e}-05$ \\
\hline \multirow{2}{*}{$f_{s i s}$} & -3.49995 & -2.33863 & -2.45923 & -3.49985 \\
& $6.58779 \mathrm{e}-06$ & .00785 & 0.00067 & $3.20262 \mathrm{e}-05$ \\
\hline \multirow{2}{*}{$f_{s t}$} & 0.0 & 0.0 & 0.0 & 0.0 \\
& 0.0 & 0.0 & 0.0 & 0.0 \\
\hline \multirow{2}{*}{$f_{S W F}$} & 0.00072 & 0.00081 & 0.00075 & 0.00073 \\
& $7.56772 \mathrm{e}-06$ & $9.5201 \mathrm{e}-05$ & $6.24289 \mathrm{e}-05$ & $2.61436 \mathrm{e}-05$ \\
\hline \multirow{2}{*}{} & & & & \\
\hline
\end{tabular}


Table 4. DE versus HDE (for $\alpha=0.5,0.05,0.005$ ) in terms of average number of functions evaluations (NFE) and average CPU time taken (in seconds) to obtain an accuracy of $10^{-4}$ for unconstrained benchmark problems of dimension 10 .

\begin{tabular}{|c|c|c|c|c|c|c|c|c|}
\hline \multirow{2}{*}{ Fun } & \multicolumn{2}{|c|}{ DE } & \multicolumn{2}{c|}{$\operatorname{HDE}(\alpha=0.5)$} & \multicolumn{2}{c|}{$\operatorname{HDE}(\alpha=0.05)$} & \multicolumn{2}{c|}{$\operatorname{HDE}(\alpha=0.005)$} \\
\cline { 2 - 9 } & NFE & Time & NFE & Time & NFE & Time & NFE & Time \\
\hline$f_{\text {SP }}$ & 14300 & 0.30 & $\mathbf{8 4 1 5}$ & 0.20 & 8835 & 0.20 & 10740 & 0.20 \\
\hline$f_{\text {ACK }}$ & 26680 & 0.60 & $\mathbf{1 3 6 9 0}$ & 0.30 & 16595 & 0.40 & 19885 & 0.50 \\
\hline$f_{R G}$ & 96400 & 2.20 & $\mathbf{8 9 3 4 5}$ & 2.10 & 92090 & 2.20 & 95120 & 2.20 \\
\hline$f_{R B}$ & $10^{6}$ & - & $10^{6}$ & - & 208190 & 10.60 & 316155 & 17.90 \\
\hline$f_{G W}$ & 94690 & 2.40 & $10^{6}$ & - & 88400 & 2.30 & 87290 & 2.20 \\
\hline$f_{L M 2}$ & 17540 & 0.90 & $\mathbf{1 0 1 7 2}$ & 0.44 & 13185 & 0.70 & 14325 & 0.70 \\
\hline$f_{p p}$ & 14290 & 0.70 & $\mathbf{7 7 6 0}$ & 0.40 & 9400 & 0.40 & 11545 & 0.60 \\
\hline$f_{\text {sis }}$ & 30940 & 0.20 & 31300 & 0.20 & $\mathbf{2 4 3 9 5}$ & 0.20 & 27695 & 0.20 \\
\hline$f_{s t}$ & 10980 & 0.50 & $\mathbf{1 1 0 3 0}$ & 0.30 & 11300 & 0.30 & 10690 & 0.30 \\
\hline$f_{S W F}$ & 25850 & 0.20 & $\mathbf{1 9 4 8 0}$ & 0.10 & 21440 & 0.20 & 22570 & 0.10 \\
\hline
\end{tabular}

Table 5. DE versus HDE (for $\alpha=0.5,0.05,0.005$ ) in terms of average number of functions evaluations (NFE) taken to obtain accuracy of $10^{-4}$ for unconstrained benchmark problems of dimension 30 .

\begin{tabular}{|c|c|c|c|c|}
\hline \multirow{2}{*}{ Fun } & \multicolumn{5}{|c|}{ NFE } \\
\cline { 2 - 5 } & $\mathrm{DE}$ & $\mathrm{HDE}(\alpha=0.5)$ & $\mathrm{HDE}(\alpha=0.05)$ & $\mathrm{HDE}(\alpha=0.005)$ \\
\hline$f_{\mathrm{SP}}$ & 146400 & $\mathbf{9 0 1 9 5}$ & 90270 & 111465 \\
\hline$f_{\mathrm{ACK}}$ & 256710 & $\mathbf{1 2 2 6 1 0}$ & 152310 & 187530 \\
\hline$f_{R G}$ & $10^{6}$ & $10^{6}$ & $10^{6}$ & $10^{6}$ \\
\hline$f_{R B}$ & $10^{6}$ & $10^{6}$ & $10^{6}$ & $10^{6}$ \\
\hline$f_{G W}$ & 222540 & $\mathbf{9 0 1 5 0}$ & 140583 & 186255 \\
\hline$f_{L M 2}$ & 182970 & $\mathbf{1 0 4 9 5 0}$ & 131325 & 149370 \\
\hline$f_{p p}$ & 225900 & $\mathbf{1 4 9 5 5 0}$ & 171150 & 190350 \\
\hline$f_{s i s}$ & 549675 & 443250 & 421650 & 429000 \\
\hline$f_{s t}$ & 126060 & 125610 & $\mathbf{1 2 1 9 5 0}$ & 125580 \\
\hline$f_{S W F}$ & 369450 & $\mathbf{2 8 3 3 9 5}$ & 305325 & 324120 \\
\hline
\end{tabular}

the parameter $\alpha$, then once again from the average fitness function value we cannot conclude anything concrete because the numerical results are more or less similar for all values of $\alpha$, which are similar to the basic DE results, which in turn are in the vicinity of the true minima. However, from Table 3, we can see that when the value of $\alpha$ is 0.5 , we get the best results in terms of NFE and CPU time with an average improvement of around $23 \%$ in case of NFE and CPU time. However, for other values of $\alpha$ also the results are better than the basic DE algorithm. Performance graphs of few selected functions for different values of $\alpha$ are given in Fig. 1(a) and 1(b). 


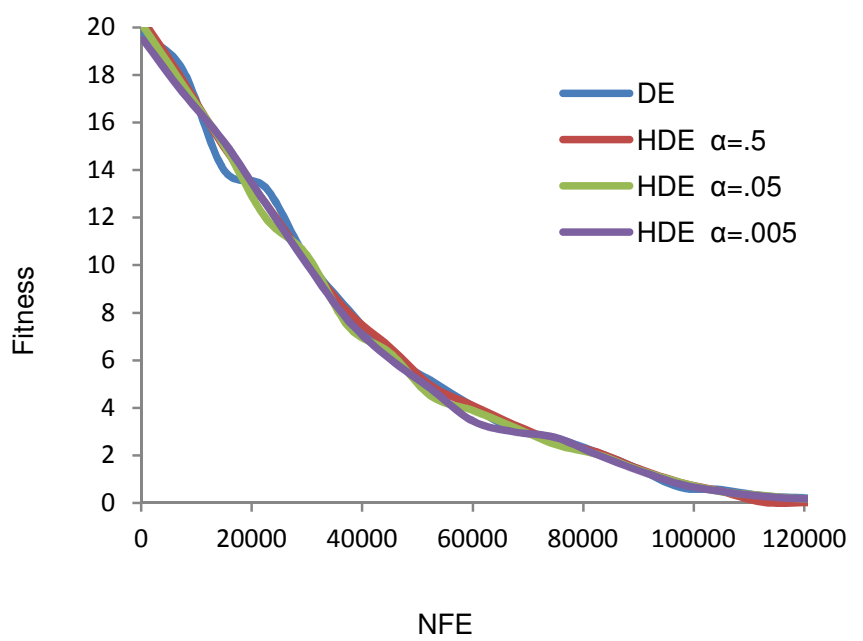

(a)

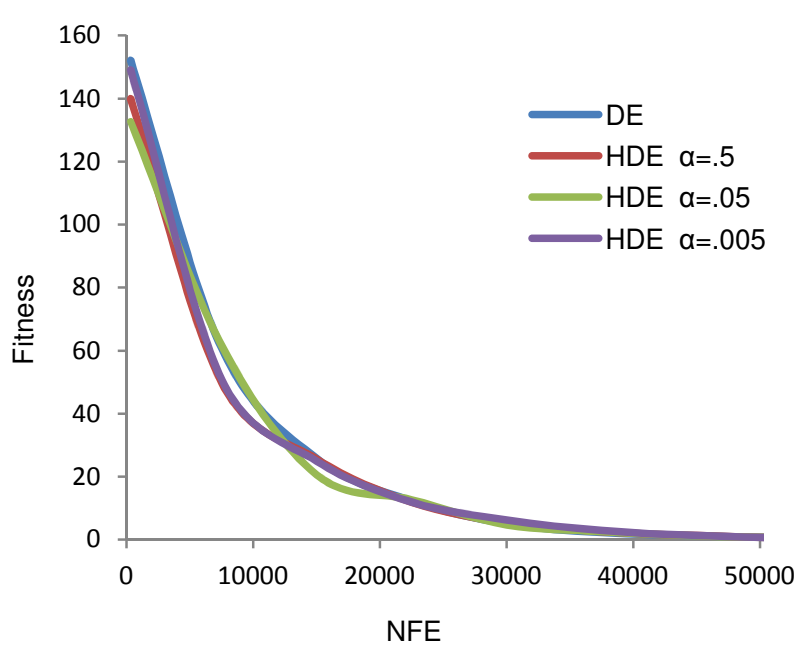

(b)

Fig. 1. Performance graph of (a) Ackley's function and (b) Sphere function.

\subsection{Numerical results for constrained real life problems}

In case of real life problems also, the proposed HDE performed better than the basic DE algorithm and the results available in literature, ${ }^{6,13,15,20,48}$ for all the four test cases. Once again the superior performance of HDE algorithm is more evident from the NFE it takes to reach the optimum solution. In terms of other performance measures also like average fitness function value and average CPU time, the proposed HDE outperformed the other algorithms. The corresponding results of real life problems are given in Tables 6 and 7 . Performance curves of real life problems are given in Figs. 2-5. 
Table 6. A comparison of numerical results of PVD and T/CSD problems using DE, HDE $(\alpha=0.05)$ and other algorithms available in literature in terms of Average Fitness function value, Number of function evaluations (NFE) Standard Deviation (std) and Time (in sec).

\begin{tabular}{|c|c|c|c|c|c|c|}
\hline \multicolumn{7}{|c|}{ Pressure vessel design (PVD) } \\
\hline Item & $\mathrm{DE}$ & $\operatorname{HDE}(\alpha=0.05)$ & SiC-PSO $^{13}$ & $\mathrm{iGSO}^{48}$ & Coello $^{15}$ & ${\text { Gene } \mathrm{AS}^{20}}^{20}$ \\
\hline$x_{1}$ & 0.778169 & 0.778169 & 0.812500 & 0.812500 & 0.812500 & 0.9375 \\
\hline$x_{2}$ & 0.384649 & 0.384649 & 0.437500 & 0.437500 & 0.437500 & 0.5000 \\
\hline$x_{3}$ & 40.3196 & 40.3197 & 42.098445 & 42.098446 & 40.3239 & 48.3290 \\
\hline$x_{4}$ & 200 & 200 & 176.636595 & 176.636596 & 200 & 112.6790 \\
\hline$g_{1}(X)$ & $-5.59078 \mathrm{e}-10$ & $-3.73187 \mathrm{e}-010$ & $-4.500 \mathrm{e}-15$ & $-3.40 \mathrm{e}-10$ & -0.034324 & -0.004750 \\
\hline$g_{2}(X)$ & $-3.0915 \mathrm{e}-009$ & $-7.13005 \mathrm{e}-011$ & -0.035880 & -0.035881 & -0.052847 & -0.038941 \\
\hline$g_{3}(X)$ & -0.0022058 & $-7.34673 \mathrm{e}-005$ & $-1.164 \mathrm{e}-10$ & -0.000029 & -27.105845 & -3652.8738 \\
\hline$g_{4}(X)$ & -40 & -40.0005 & -63.363404 & -63.363404 & -40.0000 & -127.321000 \\
\hline$f(X)$ & 5885.3301 & $\mathbf{5 8 8 5 . 3 3 0 0}$ & 6059.714335 & 6059.71400 & 6288.7445 & 6410.3811 \\
\hline$N F E$ & 40740 & 31915 & NA & 26000 & NA & NA \\
\hline Std & $1.25551 \mathrm{e}-05$ & $2.57304 \mathrm{e}-4$ & NA & NA & NA & NA \\
\hline Time $(\mathrm{sec})$ & 0.5 & 0.3 & NA & 12.34 & NA & NA \\
\hline \multicolumn{7}{|c|}{ Spring design (T/CSD) } \\
\hline Item & $\mathrm{DE}$ & $\operatorname{HDE}(\alpha=0.05)$ & ${\mathrm{SiC}-\mathrm{PSO}^{13}}^{13}$ & $\mathrm{iGSO}^{48}$ & Coello $^{15}$ & Arora $^{6}$ \\
\hline$x_{1}$ & 0.0514312 & 0.0518374 & 0.051583 & 0.051691 & 0.051480 & 0.053396 \\
\hline$x_{2}$ & 0.350492 & 0.360296 & 0.354190 & 0.356765 & 0.351661 & 0.399180 \\
\hline$x_{3}$ & 11.6701 & 11.0822 & 11.438675 & 11.286172 & 11.632201 & 9.185400 \\
\hline$g_{1}(X)$ & -0.00038971 & $-1.11544 \mathrm{e}-007$ & $-2.000 \mathrm{e}-16$ & $-2.0950 \mathrm{e}-11$ & -0.002080 & 0.000019 \\
\hline$g_{2}(X)$ & -0.00012457 & $-3.36314 \mathrm{e}-010$ & $-1.000 \mathrm{e}-16$ & $-1.3020 \mathrm{e}-11$ & -0.000110 & -0.000018 \\
\hline$g_{3}(X)$ & -4.03868 & -4.06079 & -4.048765 & -4.053880 & -4.026318 & -4.123842 \\
\hline$g_{4}(X)$ & -0.732051 & -0.725244 & -0.729483 & -0.727696 & -0.731239 & -0.698283 \\
\hline$f(X)$ & 0.0126737 & 0.0126656 & 0.012665 & 0.012665 & 0.0127048 & 0.127302737 \\
\hline$N F E$ & 9370 & 4945 & NA & 8000 & NA & NA \\
\hline Std & $4.05487 \mathrm{e}-005$ & $1.77850 \mathrm{e}-04$ & NA & NA & NA & NA \\
\hline Time (sec) & 0.2 & 0.1 & NA & 8.17 & NA & NA \\
\hline
\end{tabular}

Table 7. A comparison of numerical results of SRD and WBD problems using DE, HDE $(\alpha=0.05)$ and other algorithms available in literature in terms of Average Fitness function value, Number of function evaluations (NFE) Standard Deviation (std) and Time (in sec).

\begin{tabular}{|c|c|c|c|}
\hline \multicolumn{5}{|c|}{ Speed Reducer (SRD) } \\
\hline Item & DE & HDE $(\alpha=0.05)$ & SiC-PSO $^{13}$ \\
\hline$x_{1}$ & 3.5 & 3.5 & 3.500000 \\
\hline$x_{2}$ & 0.7 & 0.7 & 0.700000 \\
\hline$x_{3}$ & 17 & 7.30012 & 17.00000 \\
\hline$x_{4}$ & 7.3 & 7.8 & 7.30000 \\
\hline$x_{5}$ & 7.8 & 3.35021 & 7.80000 \\
\hline$x_{6}$ & 3.35021 & 5.28668 & 3.350214 \\
\hline$x_{7}$ & 5.28668 & -0.0739153 & 5.286683 \\
\hline$g_{1}(X)$ & -0.0739153 & -0.197999 & -0.073915 \\
\hline$g_{2}(X)$ & -0.197999 & -0.499148 & -0.197998 \\
\hline$g_{3}(X)$ & -0.499172 & -0.901472 & -0.499172 \\
\hline$g_{4}(X)$ & -0.901472 & $-1.64302 \mathrm{e}-009$ & -0.901471 \\
\hline$g_{5}(X)$ & $-2.381 \mathrm{e}-008$ & $-2.97741 \mathrm{e}-010$ & 0.000000 \\
\hline$g_{6}(X)$ & $-1.66315 \mathrm{e}-009$ & -0.7025 & $-5.000 \mathrm{e}-16$ \\
\hline$g_{7}(X)$ & -0.7025 & $-4.98685 \mathrm{e}-011$ & -0.702500 \\
\hline$g_{8}(X)$ & $-3.07603 \mathrm{e}-010$ & -0.583333 & $-1.000 \mathrm{e}-16$ \\
\hline$g_{9}(X)$ & -0.583333 & -0.0513413 & -0.583333 \\
\hline$g_{10}(X)$ & -0.0513257 & -0.0108524 & 0.051325 \\
\hline$g_{11}(X)$ & -0.0108524 & $\mathbf{2 9 9 6 . 3 4 7 1 0 1}$ & -0.010852 \\
\hline$f(X)$ & 2996.35 & $\mathbf{7 8 6 7 . 5}$ & 2996.348165 \\
\hline$N F E$ & 8925 & $\mathbf{1 . 3 5 4 6 1 e - 0 3}$ & NA \\
\hline Std & $9.02658 \mathrm{e}-06$ & $\mathbf{0 . 3}$ & NA \\
\hline$T i m e(s e c)$ & 0.4 & & NA \\
\hline & & & \\
\hline
\end{tabular}


Table 7. (Continued)

\begin{tabular}{|c|c|c|c|}
\hline \multicolumn{4}{|c|}{ Welded Beam Design(WBD) } \\
\hline Item & DE & HDE $(\alpha=0.05)$ & SiC-PSO $^{13}$ \\
\hline$x_{1}$ & 0.205727 & 0.182634 & 0.205729 \\
\hline$x_{2}$ & 3.47055 & 3.81039 & 3.470488 \\
\hline$x_{3}$ & 9.03667 & 9.58501 & 9.036624 \\
\hline$x_{4}$ & 0.20573 & 0.182863 & 0.205729 \\
\hline$g_{1}(X)$ & -0.0681061 & -0.00693705 & $-1.819 \mathrm{e}-12$ \\
\hline$g_{2}(X)$ & -0.458633 & -0.14485 & -0.003721 \\
\hline$g_{3}(X)$ & $-3.57774 \mathrm{e}-06$ & -0.000228855 & 0.00000 \\
\hline$g_{4}(X)$ & -3.43296 & -3.49465 & -3.432983 \\
\hline$g_{5}(X)$ & -0.0807269 & -0.0576342 & -0.080729 \\
\hline$g_{6}(X)$ & -0.235541 & -0.236368 & -0.235540 \\
\hline$g_{7}(X)$ & -2055.41 & -0.0348941 & 0.000000 \\
\hline$f(X)$ & 1.72487 & 1.64226 & 1.724852 \\
\hline$N F E$ & 17425 & 11790 & NA \\
\hline Std & $7.27403 \mathrm{e}-05$ & $1.31468 \mathrm{e}-05$ & NA \\
\hline Time $($ sec $)$ & $0.4 \mathrm{sec}$ & 0.3 & NA \\
\hline
\end{tabular}

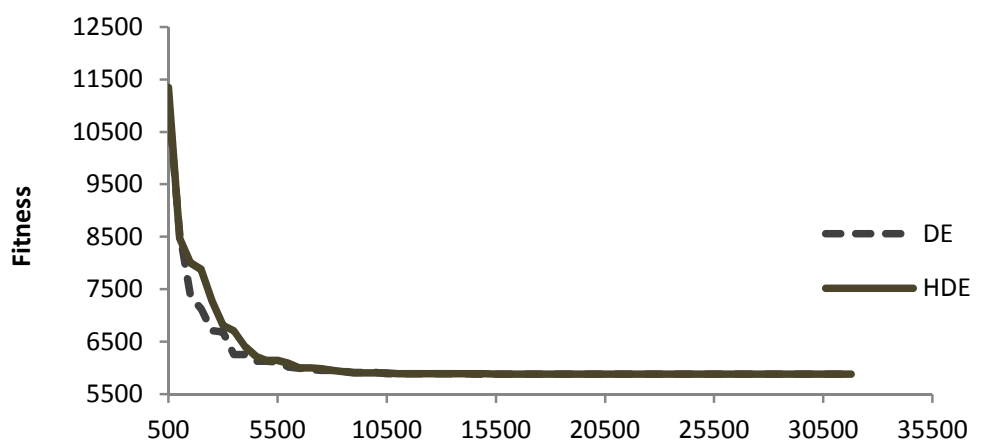

Number of function evaluation

Fig. 2. Convergence graph of DE and HDE for pressure vessel design problem.

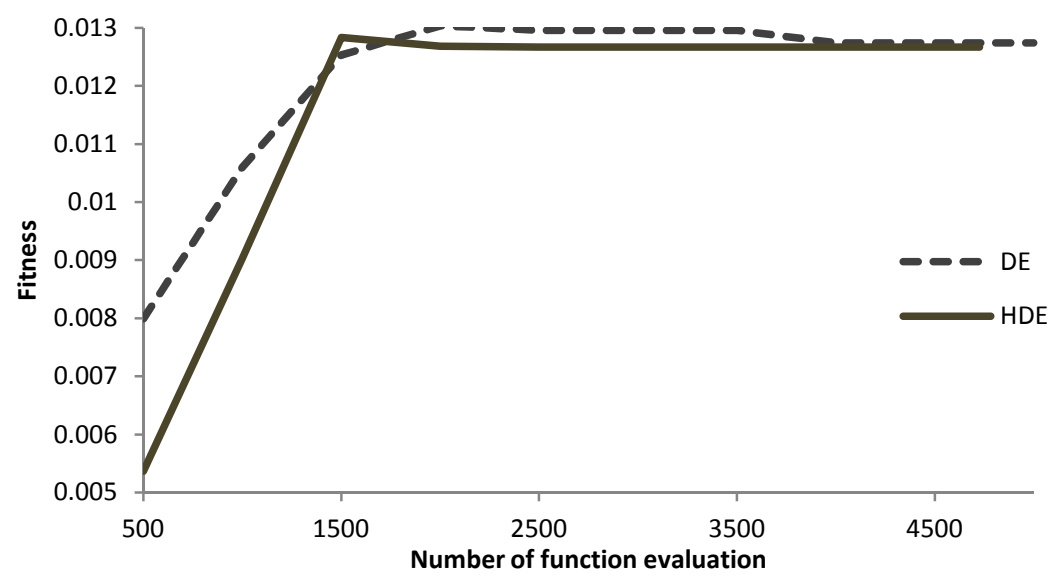

Fig. 3. Convergence graph of DE and HDE for spring design problem. 


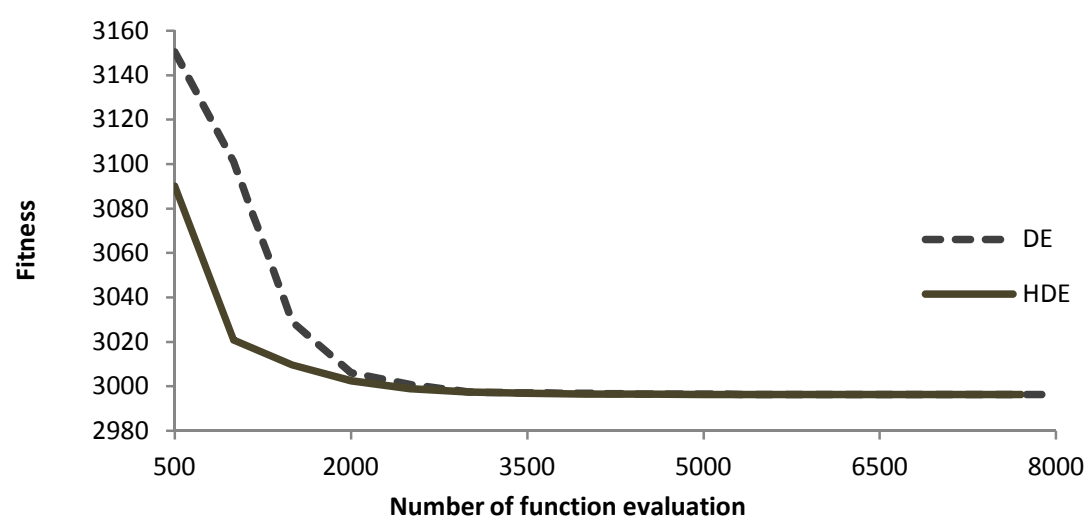

Fig. 4. Convergence graph of DE and HDE for Speed reducer design problem.

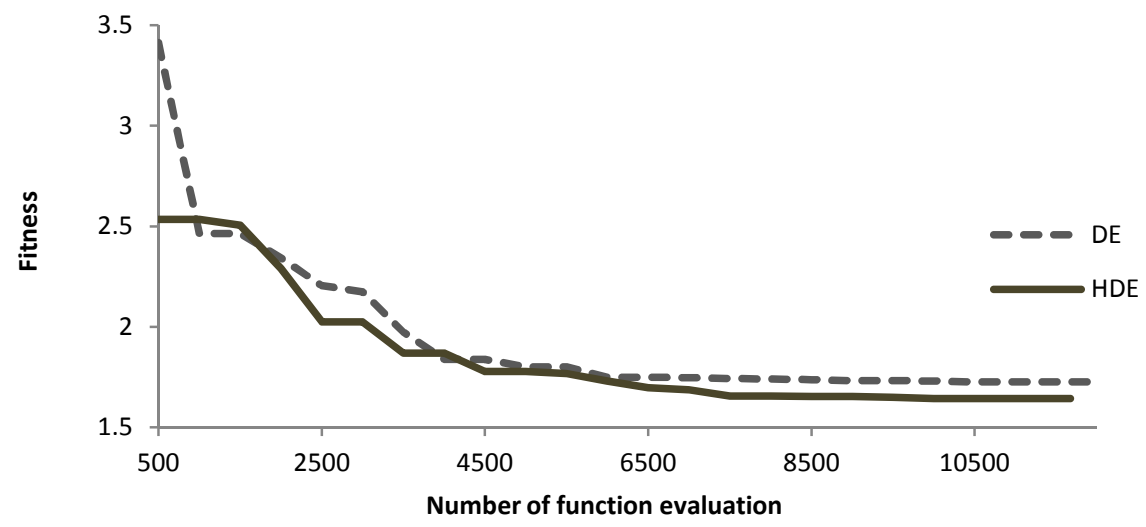

Fig. 5. Convergence graph of DE and HDE for welded beam design problem.

\section{Conclusions}

In the present study, we proposed a simple and easy to implement hybrid version of DE and PSO algorithm called HDE. The proposed HDE algorithm works in two stages. In the first stage DE is activated to contract the search space and when the search space has contracted enough, PSO is activated to further refine the search. The swap from DE to PSO is decided with the help of a switchover constant named $\alpha$. We tested the proposed algorithm for scalable problems of dimensions 10 and 30 and observed that although it does not make much improvement in the fitness function value but in terms of NFE and CPU time there is an improvement of more than 20\%. The superior performance of HDE is also evident from the four cases of real life problems, where it outperformed the other algorithms in terms of all the performance measures taken for the present study. We would also like to point out that we have used the basic versions of DE 
and PSO. It would be interesting to note the performance of the proposed scheme while using some more advanced versions of DE and PSO algorithms.

\section{References}

1. H. Abbass, The self-adaptive pareto differential evolution algorithm, Proceedings of the 2002 Congress on Evolutionary Computation, pp. 831-836, 2002.

2. M. A. Abido, Optimal design of power system stabilizers using particle swarm optimization, IEEE Transactions on Energy Conversion 17(3), 406-413, 2002.

3. A. Abraham, S. Das, and A. Konar, Kernel based automatic clustering using modified particle swarm optimization algorithm, 2007 Genetic and Evolutionary Computation Conference, GECCO 2007, ACM Press, Dirk Thierens et al. (Eds.), ISBN 978-1-59593-698-1, pp. 2-9, 2007.

4. A. Abraham, H. Liu, and T. G. Chang, Variable neighborhood particle swarm optimization algorithm, Genetic and Evolutionary Computation Conference (GECCO-2006), Seattle, USA, Late Breaking Papers, CD Proceedings, Jörn Grahl (Ed.), 2006.

5. M. M. Ali, Differential evolution with preferential crossover, European Journal of Operation Research 181, pp. 1137-1147, 2007.

6. J. S. Arora, Introduction to Optimum Design, New York, McGrow-Hill, 1989.

7. T. Back, F. Hoffmeister, and H. Schwefel, A survey of evolution strategies. In: Proceedings of the Fourth International Conference on Genetic Algorithms and their Applications, pp. 2-9, 1991.

8. C. S. Beightler, D. Phillips, and D. Wilde, Foundations of Optimization (Prentice-Hall, Englewood Cliffs, NJ: 1979), 2nd edn.

9. K. Bergey Paul and Cliff Ragsdale, Modified differential evolution: A greedy random strategy for genetic recombination, Omega The International Journal of Management Science 33, 255-265, 2005.

10. V. D. F. Bergh, Particle swarm weight initialization in multi-layer perceptron artificial neural networks, Development and Practice of Artificial Intelligence Techniques (Durban, South Africa, 1999), pp. 41-45.

11. T. R. Bhat, D. Venkataramani, V. Ravi, and C. V. S. Murty, An improved differential evolution method for efficient parameter estimation in biofilter modeling, Biochemical Engineering Journal 28, 167-176, 2006.

12. J. Brest, S. Greiner, B. Boškovic, M. Mernik, and V. Žumer, Self-adapting control parameters in differential evolution: A comparative study on numerical benchmark problems, IEEE Transactions on Evolutionary Computation, Vol. 10, Issue 6, pp. 646-657, 2006.

13. L. C. Cagnina, S. C. Esquivel, and C. A. Coello Coello, Solving engineering optimization problems with the simple constrained particle optimizer, Informatica 32, 319-326, 2008.

14. Ji-Pyng, Chiou Chung-Fu Chang, and Ching-Tzong Su, Ant direction hybrid differential evolution for solving large capacitor placement problems, IEEE Transactions on power systems, 19(4), 1794-1800, 2004.

15. C. A. Coello Coello, Use of a self adaptive penalty approach for engineering optimization problems, Computer in Industry 41(2), 113-127, 2000.

16. S. Das and A. Konar, Two-dimensional IIR filter design with modern search heuristics: A comparative study, International Journal of Computational Intelligence and Applications 6(3), Imperial College Press, 2006.

17. S. Das and A. Konar, A swarm intelligence approach to the synthesis of two-dimensional IIR filters, Engineering Applications of Artificial Intelligence 20(8), 1086-1096, http://dx.doi.org/ 10.1016/j.engappai.2007.02.004, 2007. 
18. S. Das, A. Abraham, and A. Konar, Adaptive clustering using improved differential evolution algorithm, IEEE Transactions on Systems, Man and Cybernetics - Part A, IEEE Press, New York, USA, 2007.

19. S. Das, A. Konar, and U. K. Chakraborty, Two improved differential evolution schemes for faster global search, ACM-SIGEVO Proceedings of GECCO, Washington D.C., pp. 991-998, 2005.

20. K. Deb, GeneAS: A robust optimal design technique for mechanical component design, in D. Dasgupta and Z. Michalewicz (Eds.), Evolutionary Algorithms in Engineering Applications (Berlin, Springer-Verlage, 1997), pp. 497-514.

21. K. Deb, An efficient constraint handling method for genetic algorithms, Computer Methods in Applied Mechanics and Engineering 186(2/4):311-338, 2000.

22. S. Doyle, D. Corcoran, and J. Connell, Automated mirror design using an evolution strategy, Optical Engineering 38(2), 323-333, 1999.

23. R. C. Eberhart and X. Hu, Human tremor analysis using particle swarm optimization. In: Proceedings of the IEEE Congress on Evolutionary Computation (CEC 1999), Washington D.C., pp. 1927-1930, 1999.

24. L. Fogel, Evolutionary programming in perspective: The top-down view. In: J. M. Zurada, R. Marks, Jr., and C. Robinson (Eds.), Computational Intelligence: Imitating Life (IEEE Press, Piscataway, NJ, USA, 1994).

25. D. Goldberg, Genetic Algorithms in Search Optimization and Machine Learning (AddisonWesley, 1989).

26. Z. He, C. Wei, L. Yang, X. Gao, S. Yao, R. C. Eberhart, and Y. Shi, Extracting rules from fuzzy neural network by particle swarm optimization. In: Proceedings of IEEE Congress on Evolutionary Computation (CEC 1998), Anchorage, Alaska, USA, 1998.

27. T. Hendtlass, A combined swarm differential evolution algorithm for optimization problems. In: Proceedings of the Fourteenth International Conference on Industrial and Engineering Applications of Artificial Intelligence and Expert Systems. Lecture Notes in Computer Science, Vol. 2070. Springer-Verlag, pp. 11-18, 2001.

28. E. Himmelblau and Lasdon, Optimization of Chemical Processes (McGraw Hill, 1972), 2nd edn.

29. Hui-Yuan Fan and Jouni Lampinen, A trigonometric mutation operation to differential evolution, Journal of Global Optimization 27:105-129, 2003.

30. T, Jayabarathi Sandeep Chalasani, Zameer Ahmed Shaik, and Nishchal Deep Kodali, Hybrid differential evolution and particle swarm optimization based solutions to short term hydro thermal scheduling, WSEAS Transactions on Power Systems 11(2), 245-254, 2007.

31. P. Kaelo and M. M. Ali, Differential evolution algorithm with hybrid mutation, Compt. Optim. Appl. 37, 231-246, 2007.

32. S. Kannan, S. Slochanal, P. Subbaraj, and N. Padhy, Application of particle swarm optimization technique and its variants to generation expansion planning, Electric Power Systems Research 70(3), 203-210, 2004.

33. J. Kennedy and R. C. Eberhart, Particle swarm optimization, IEEE Int. Conf. on Neural Networks (Perth, Australia), IEEE Service Center, Piscataway, NJ, pp. 1942-1948, 1995.

34. J. Lampinen and I. Zelinka, On stagnation of the differential evolution algorithm, in: Pavel Ošmera (Ed.), Proceedings of MENDEL 2000, 6th International Mendel Conference on Soft Computing, pp. 76-83, Brno, Czech Republic, 2000.

35. T. Masters and W. Land, A new training algorithm for the general regression neural network, Proceedings of Computational Cybernetics and Simulation, organized by IEEE Systems, Man, and Cybernetics Society 3, 1990-1994, 1997. 
36. L. Messerschmidt and A. P. Engelbrecht, Learning to play games using a PSO-based competitive learning approach, IEEE Transactions on Evolutionary Computation 8(3), 280288, 2004.

37. M. Omran, A. Salman, and A. P. Engelbrecht, Self-adaptive differential evolution, computational intelligence and security, PT 1, Proceedings Lecture Notes in Artificial Intelligence 3801: 192-199, 2005.

38. M. G. H. Omran, A. P. Engelbrecht, and A. Salman, Bare bones differential evolution, European Journal of Operational Research, doi:10.1016/j.ejor.2008.02.035, 2008.

39. M. Pant, M. Ali, and V. P. Singh, Differential evolution with parent centric crossover, Second UKSIM European Symposium on Computer Modeling and Simulation, 141-146, 2008.

40. M. Pant, M. Ali, and V. P. Singh, Parent centric differential evolution algorithm for global optimization, OPSEARCH 46(2), 153-168, 2009.

41. S. Paterlini and T. Krink, Differential evolution and particle swarm optimization in partitional clustering, Computational Statistics and Data Analysis, Vol. 50, 1220-1247, 2006.

42. S. Rahnamayan, H. R. Tizhoosh, and M. M. A. Salama, A novel population initialization method for accelerating evolutionary algorithms, Computer and Applied Mathematics with Application (53), 1605-1614, 2007.

43. S. S. Rao, Engineering Optimization: Theory and Practice (Wiley Eastern Ltd., 1996), 3rd edn.

44. T. Rogalsky, S. Kocabiyik, and R. Derksen, Differential evolution in aerodynamic optimization, Canadian Aeronautics and Space Journal 46(4), 183-190, 2000.

45. J. Salerno, Using the particle swarm optimization technique to train a recurrent neural model, IEEE International Conference on Tools with Artificial Intelligence, pp. 45-49, 1997.

46. A. Salman, A. P. Engelbrecht, and M. G. H. Omran, Empirical analysis of self adaptive differential evolution, European Journal of Operational Research 183, pp. 785-804, 2007.

47. E. Sandgren, Nonlinear integer and discrete programming in mechanical design optimization, J. Mech. Des.-T. ASME 112(2):223-229, 1990.

48. H. Shen, Y. Zhu, B. Niu, and Q. H. Wu, An improved group search optimizer for mechanical design optimization problems, Progress in Natural Science 19, 91-97, 2009.

49. Y. Shi and R. C. Eberhart, A modified particle swarm optimiser, IEEE International Conference on Evolutionary Computation, Anchorage, Alaska, May 4-9, 1998.

50. Y. Shi and R. C. Eberhart, Fuzzy adaptive particle swarm optimization, In: Proceedings of the Congress on Evolutionary Computation 2001, Seoul, Korea, IEEE Service Center, IEEE (2001), pp. 101-106, 2001.

51. R. Storn and K. Price, Differential evolution - A simple and efficient adaptive scheme for global optimization over continuous spaces, Technical Report TR-95-012, Berkeley, CA, 1995.

52. G. Stumberger, D. Dolinar, U. Pahner, and K. Hameyer, Optimization of radial active magnetic bearings using the finite element technique and differential evolution algorithm, IEEE Transactions on Magnetics 36(4), 1009-1013, 2000.

53. H. Talbi and M. Batouche, Hybrid particle swarm with differential evolution for multimodal image registration. In: Proceedings of the IEEE International Conference on Industrial Technology, Vol. 3, pp. 1567-1573, 2004.

54. M. P. Wachowiak, R. Smolikova, Y. Zheng, M. J. Zurada, and A. S. Elmaghraby, An approach to multimodal biomedical image registration utilizing particle swarm optimization, IEEE Transactions on Evolutionary Computation, 8(3), 289-301, 2004.

55. F. S. Wang and J. W. Sheu, Multi-objective parameter estimation problems of fermentation processes using high ethanol tolerance yeast, Chemical Engineering Science 55(18), 36853695, 2000. 
56. W. Xu and X. Gu, A hybrid particle swarm optimization approach with prior crossover differential evolution. In: Proceedings of GEC09 pp. 671-677, 2009.

57. Z. Yang, J. He, and X. Yao, Making a difference to differential evolution, in Z. Michalewicz and P. Siarry (Eds.), Advances in Metaheuristics for Hard Optimization (Springer, 2007), pp. 415-432.

58. H. Yoshida, K. Kawata, Y. Fukuyama, S. Takayama, and Y. Nakanishi, A particle swarm optimization for reactive power and voltage control considering voltage security assessment, IEEE Transactions on Power Systems 15(4), 1232-1239, 2000.

59. D. Zaharie, Control of population diversity and adaptation in differential evolution algorithms, In D. Matousek and P. Osmera (Eds.), Proc. of MENDEL 2003, 9th International Conference on Soft Computing, Brno, Czech Republic, pp. 41-46, 2003.

60. I. Zelinka and J. Lampinen, An evolutionary learning algorithms for neural networks. In: Proceedings of Fifth International Conference on Soft Computing, MENDEL'99, pp. 410-414, 1999.

61. C. Zhang, J. Ning, S. Lu, D. Ouyang, and T. Ding, A novel hybrid differential evolution and particle swarm optimization algorithm for unconstrained optimization, Operations Research Letters, Vol. 37, 117-122, 2009.

62. W. J. Zhang and X. F. Xie, DEPSO: Hybrid particle swarm with differential evolution operator. In: IEEE International Conference on Systems, Man, and Cybernetics, Vol. 4, pp. 3816-3821, 2003.

63. Z.-F. Hao, G.-H. Gua, and H. Huang, A particle swarm optimization algorithm with differential evolution, Proceedings of Sixth Int. Conf. on Machine Learning and Cybernetics, pp. 1031-1035, 2007.

64. G.-N. Jose, E. Alba, and J. Apolloni, Particle swarm hybridized with differential evolution: black box optimization benchmarking for noisy functions, Proceedings of Int. Conf. Genetic and Evolutionary Computation, pp. 2343-2350, 2009.

65. H. Liu, Z. Cai, and Y. Wang, Hybridizing particle swarm optimization with differential evolution for constrained numerical and engineering optimization, Applied Soft Computing, 10:629-640 (2009).

66. Caponio, F. Neri, and V. Tirronen, Superfit control adaption in memetic differential evolution frameworks, Soft Computing, 13:811-831(2009).

67. W. Xu and $\mathrm{X}$. Gu, A hybrid particle swarm optimization approach with prior crossover differential evolution, Proceedings of ACM/SIGEVO summit on Genetic and Evolutionary Computation, pp. 671-678, 2009.

68. M. Pant, R. Thangaraj, and A. Abraham, DE-PSO: A new hybrid meta-heuristic for solving global optimization problems, New Mathematics and Natural Computation, accepted for publication (2009).

69. S. Khamsawang, P. Wannakarn, and S. Jiriwibhakorn Hybrid PSO-DE for solving the economic dispatch problem with generator constraints, Proceedings of the IEEE Int. Conf. on Computer and Automation Engineering, Vol. 5, pp. 135-139, 2010. 


\section{Appendix A. Unconstrained Benchmark Problems}

1. Sphere function (SP):

$$
f_{S P}(x)=\sum_{i=1}^{n} x_{i}^{2} \text { with }-5.12 \leq x_{i} \leq 5.12, \min f_{s p}(0, \ldots, 0)=0
$$

2. Acley's function (ACK):

$$
\begin{aligned}
& f_{A C K}(X)=-20 * \exp \left(-.2 \sqrt{1 / n \sum_{i=1}^{n} x_{i}^{2}}\right)-\exp \left(1 / n \sum_{i=1}^{n} \cos \left(2 \pi x_{i}\right)\right)+20+e, \\
& \text { with }-30 \leq x_{i} \leq 30, \min f_{A C K}(0, \ldots, 0)=0 .
\end{aligned}
$$

3. Rastrigin's function $(\mathrm{RG})$ :

$$
f_{R G}(x)=10 n+\sum_{i=1}^{n}\left(x_{i}^{2}-10 \cos \left(2 \pi x_{i}\right)\right), \text { with }-5.12 \leq x_{i} \leq 5.12, \min f_{R G}(0, \ldots ., 0)=0 .
$$

4. Rosenbrock Problem (RB):

$$
f_{R B}(x)=\sum_{i=1}^{n-1}\left[100\left(x_{i+1}^{2}-x_{i}^{2}\right)+\left(1-x_{i}^{2}\right)\right], \text { with }-30 \leq x_{i} \leq 30, \min f_{R B}(1, \ldots, 1)=0 .
$$

5. Griewenk function $(\mathrm{GW})$ :

$f_{G W}(x)=\frac{1}{4000} \sum_{i=1}^{n} x_{i}^{2}-\prod_{i=1}^{n} \cos \left(\frac{x_{i}}{\sqrt{i}}\right)+1$, with $-600 \leq x_{i} \leq 600, \min f_{G W}(0, \ldots, 0)=0$.

6. Levy and Montalvo 2 Problem (LM2):

$$
\begin{aligned}
& f_{L M 2}(x)=\sin ^{2}\left(3 \pi x_{1}\right)+\sum_{i=1}^{n-1}\left(x_{i}-1\right)\left(1+\sin ^{2}\left(3 \pi x_{i+1}\right)\right)+\left(x_{n}-1\right)\left(1+\sin ^{2}\left(2 \pi x_{n}\right)\right), \\
& \text { with }-5 \leq x_{i} \leq 5, \min f_{L M 2}(1, \ldots, 1)=0 .
\end{aligned}
$$

7. Paviani problem (PP):

$$
f_{P P}(x)=\sum_{i=1}^{n}\left[\left(\ln \left(x_{i}-2\right)\right)^{2}+\left(\ln \left(10-x_{i}\right)\right)^{2}\right]-\left(\prod_{i=1}^{n} x_{i}\right)^{0.2}, \text { with } 2 \leq x_{i} \leq 10 .
$$

8. Sinusoid function (SIN):

$$
\begin{aligned}
& f_{S I N}(x)=-\left[A \prod_{i=1}^{n} \sin \left(x_{i}-z\right)+\prod_{i=1}^{n} \sin \left(B\left(x_{i}-z\right)\right)\right], \text { with } 0 \leq x_{i} \leq 180, \\
& \min f_{S I N}\left(x^{*}\right)=-(\mathrm{A}+1) \text { where } x_{i}^{*}=(90+z), A=2.5, B=5, z=30 .
\end{aligned}
$$

9. Step function (ST):

$$
f_{S T}(x)=\sum_{i=1}^{n}\left(\left\lfloor x_{i}+0.5\right\rfloor\right)^{2} \text {, with }-100 \leq x_{i} \leq 100, \min f_{S T}\left(-0.5 \leq x_{i} \leq 0.5\right)=0 .
$$

10. Schwefel's problem (SWF):

$$
f_{S W F}(x)=418.9829 \times n-\sum_{i=1}^{n} x_{i} \sin \left(\sqrt{\left|x_{i}\right|}\right),-500 \leq x_{i} \leq 500, \min f_{S W F}(s, \ldots, s)=0
$$

where $s=420.97$. 


\section{Appendix B. Real Life Problems}

\section{B.1. Welded beam design optimization problem (WBD)}

The problem is to design a welded beam for minimum cost, subject to some constraints. ${ }^{13}$ Figure 6 shows the welded beam structure which consists of a beam A and the weld required to hold it to member $B$. The objective is to find the minimum fabrication cost, considering four design variables: $x_{1}, x_{2}, x_{3}, x_{4}$ and constraints. The optimization model is summarized below:

$$
\text { Minimize: } f(X)=1.10471 x_{1}^{2} x_{2}+0.04811 x_{3} x_{4}\left(14.0+x_{2}\right)
$$

Subject to:

$$
\begin{gathered}
g_{1}(X)=\tau(X)-13,6000 \leq 0 \\
g_{2}(X)=\sigma(X)-30,000 \leq 0 \\
g_{3}(X)=x_{1}-x_{4} \leq 0 \\
g_{4}(X)=0.10471 x_{1}^{2}+0.04811 x_{3} x_{4}\left(14.0+x_{2}\right)-5.0 \leq 0 \\
g_{5}(X)=0.125-x_{1} \leq 0 \\
g_{6}(X)=\delta(X)-0.25 \leq 0 \\
g_{7}(X)=6,000-P c(X) \leq 0
\end{gathered}
$$

with:

$$
\begin{gathered}
\tau(X)=\sqrt{\left(\tau^{\prime}\right)^{2}+\left(2 \tau^{\prime} \tau^{\prime \prime}\right) \frac{x_{2}}{2 R}+\left(\tau^{\prime \prime}\right)^{2}} \\
\tau^{\prime}(X)=\frac{6,000}{\sqrt{2} x_{1} x_{2}} \\
\tau^{\prime \prime}(X)=\frac{M R}{J} \\
M=6,000\left(14+\frac{x_{2}}{2}\right) \\
R=\sqrt{\frac{x_{2}}{2 R}+\left(\frac{x_{1}+x_{3}}{2}\right)^{2}} \\
J=2\left\{x_{1} x_{2} \sqrt{2}\left[\frac{x_{2}^{2}}{12}+\left(\frac{x_{1}+x_{3}}{2}\right)^{2}\right]\right\} \\
\sigma(X)=\frac{504,000}{x_{4} x_{3}^{2}}
\end{gathered}
$$


M. Ali et al.

$$
\begin{gathered}
\delta(X)=\frac{65,856,000}{\left(30 \times 10^{6}\right) x_{4} x_{3}^{3}} \\
\operatorname{Pc}(X)=\frac{4.013\left(30 \times 10^{6}\right) \sqrt{\frac{x_{3}^{2} x_{4}^{6}}{36}}}{196}\left(1.0-\frac{x_{3} \sqrt{\frac{30 \times 10^{6}}{4\left(12 \times 10^{6}\right)}}}{28}\right)
\end{gathered}
$$

with $0.1 \leq x_{1}, x_{4} \leq 2.0$ and $0.1 \leq x_{2}, x_{3} \leq 10.0$

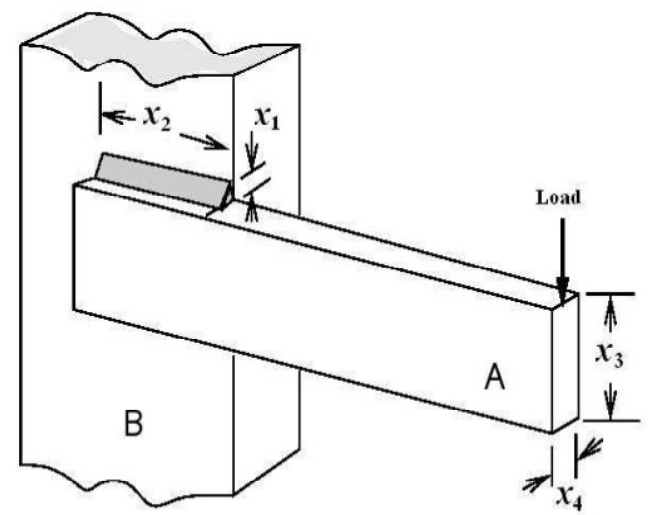

Fig. 6. Welded beam.

\section{B.2. Pressure vessel design optimization problem (PVD)}

As shown in Fig. 7, pressure vessel introduced by Sandgren ${ }^{47}$ was designed aimed at minimizing the total cost of materials, forming and welding of the pressure vessel. There are four design variables: The shell thickness $x_{1}$, the thickness of the head $x_{2}$, the inner radius $x_{3}$ and the length of the cylindrical section $x_{4}, x_{1}$ and $x_{2}$ are discrete values which are integer multiples of $0.0625 \mathrm{in}, x_{3}$ and $x_{4}$ are continuous. The pressure vessel problem is stated as follows:

Minimize: $f(X)=0.6224 x_{1} x_{3} x_{4}+1.7781 x_{2} x_{3}^{2}+3.1661 x_{1}^{2} x_{4}+19.84 x_{1}^{2} x_{3}$

Subject to:

$$
\begin{gathered}
g_{1}(X)=0.0193 x_{3}-x_{1} \leq 0 \\
g_{2}(X)=0.00954 x_{3}-x_{2} \leq 0 \\
g_{3}(X)=1,296,000-\pi x_{3}^{2} x_{4}^{2}-\frac{4}{3} \pi x_{3}^{3} \leq 0 \\
g_{4}(X)=x_{4}-240 \leq 0
\end{gathered}
$$

with $0.0625 \leq x_{1}, x_{2} \leq 99 \times 0.0625$ and $10.0 \leq x_{3}, x_{4} \leq 200.0$. 

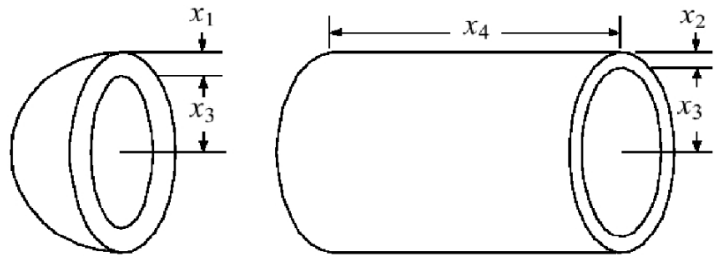

Fig. 7. Pressure vessel.

\section{B.3. Speed reducer design optimization problem (SRD)}

The design of the speed reducer ${ }^{13}$ shown in Fig. 8, is considered with the face width $x_{1}$, module of teeth $x_{2}$, number of teeth on pinion $x_{3}$, length of the first shaft between bearings $x_{4}$, length of the second shaft between bearings $x_{5}$, diameter of the first shaft $x_{6}$, and diameter of the first shaft $x_{7}$ (all variables continuous except $x_{3}$ that is integer). The weight of the speed reducer is to be minimized subject to constraints on bending stress of the gear teeth, surface stress, transverse deflections of the shafts and stresses in the shaft. The problem is:

$$
\text { Minimize: } \begin{aligned}
f(X)= & 0.7854 x_{1} x_{2}^{2}\left(3.3333 x_{3}^{2}+14.9334 x_{3}-43.0934\right) \\
& -1.508 x_{1}\left(x_{6}^{2}+x_{7}^{2}\right)+7.4777\left(x_{6}^{3}+x_{7}^{3}\right) \\
& +0.7854\left(x_{4} x_{6}^{2}+x_{5} x_{7}^{2}\right)
\end{aligned}
$$

Subject to:

$$
\begin{gathered}
g_{1}(X)=\frac{27}{x_{1} x_{2}^{2} x_{3}}-1.0 \leq 0 \\
g_{2}(X)=\frac{397.5}{x_{1} x_{2}^{2} x_{3}^{2}}-1.0 \leq 0 \\
g_{3}(X)=\frac{1.93 x_{4}^{3}}{x_{2} x_{3} x_{6}^{4}}-1.0 \leq 0 \\
g_{5}(X)=\frac{1.0}{110 x_{6}^{3}} \sqrt{\left(\frac{745.0 x_{4}}{x_{2} x_{3}}\right)^{2}+16.9 \times 10^{6}}-1.0 \leq 0 \leq 0 \\
g_{6}(X)=\frac{1.0}{85 x_{7}^{3}} \sqrt{\left(\frac{745.0 x_{5}}{x_{2} x_{3}}\right)^{2}+157.5 \times 10^{6}}-1.0 \leq 0 \\
g_{7}(X)=\frac{x_{2} x_{3}}{40.0}-1.0 \leq 0
\end{gathered}
$$


M. Ali et al.

$$
\begin{gathered}
g_{8}(X)=\frac{5 x_{2}}{x_{1}}-1.0 \leq 0 \\
g_{9}(X)=\frac{x_{1}}{12 x_{2}}-1.0 \leq 0 \\
g_{10}(X)=\frac{1.5 x_{6}+1.9}{x_{4}}-1.0 \leq 0 \\
g_{11}(X)=\frac{1.1 x_{7}+1.9}{x_{5}}-1.0 \leq 0
\end{gathered}
$$

with $2.6 \leq x_{1} \leq 3.6,0.7 \leq x_{2} \leq 0.8,17 \leq x_{3} \leq 28,7.3 \leq x_{4} \leq 8.3,7.8 \leq x_{5} \leq 8.3$, $2.9 \leq x_{6} \leq 3.9$ and $5.0 \leq x_{7} \leq 5.5$.

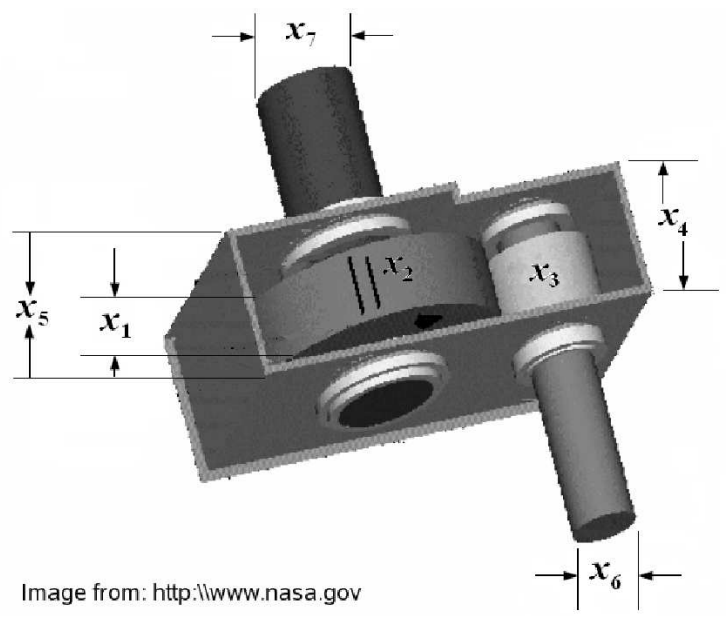

Fig. 8. Speed reducer.

\section{B.4. Tension/compression spring design optimization problem (T/CSD)}

As shown in Fig. 9, spring was designed aiming at minimizing the weight of a tension/ compression spring. ${ }^{13}$ There are three design variables: the wire diameter $x_{1}$, the mean coil diameter $x_{2}$ and the number of active coils $x_{3}$. All design variables are continuous. The spring problem is stated as follows:

$$
\text { Minimize: } f(X)=x_{1}^{2} x_{2}\left(2.0+x_{3}\right)
$$

Subject to:

$$
\begin{gathered}
g_{1}(X)=1.0-\frac{x_{2}^{3} x_{3}}{7,178 x_{1}^{4}} \leq 0 \\
g_{2}(X)=\frac{4 x_{2}^{2}-x_{1} x_{2}}{12,566 x_{2} x_{1}^{3}-x_{1}^{4}}+\frac{1}{5,108 x_{1}^{2}}-1.0 \leq 0
\end{gathered}
$$




$$
\begin{gathered}
g_{3}(X)=1.0-\frac{140.45 x_{1}}{x_{2}^{2} x_{3}} \leq 0 \\
g_{4}(X)=\frac{x_{1}+x_{2}}{1.5}-1.0 \leq 0
\end{gathered}
$$

with $0.05 \leq x_{1} \leq 2.0,0.25 \leq x_{2} \leq 1.3$ and $2.0 \leq x_{3} \leq 15.0$.

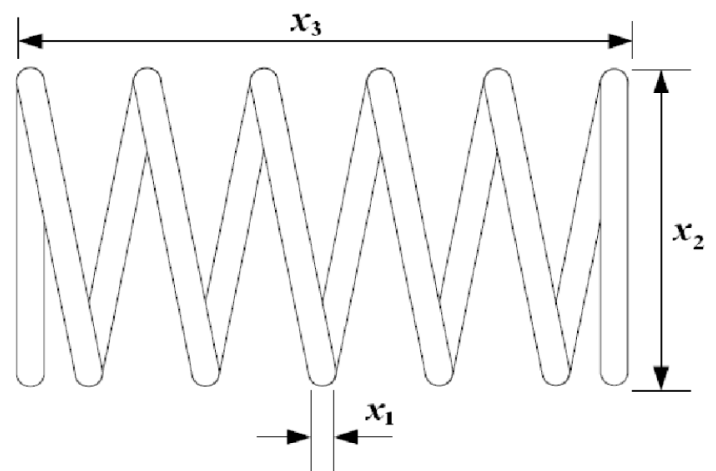

Fig. 9. Tension/compression spring. 